\title{
INVESTIGACIONES
}

\section{Investigación formativa como estrategia pedagógica: caso de estudio ingeniería industrial de la I.U Pascual Bravo}

\author{
Formative research as a pedagogical strategy: \\ industrial engineering case study of the I.U Pascual Bravo
}

\section{Iván Darío Rojas Arenas ${ }^{a}$, José Alejandro Durango Marín ${ }^{a}$, Jorge Amado Rentería Vera ${ }^{a}$}

${ }^{a}$ Institución Universitaria Pascual Bravo.

ivan.rojasar@pascualbravo.edu.co, ja.durango@pascualbravo.edu.co, j.renteriave@ pascualbravo.edu.co

\section{RESUMEN}

La generación de una cultura investigativa y la formación integral de sus profesionales es una problemática constante para las instituciones de educación superior del país; la separación entre academia y sociedad aísla a las universidades del contexto donde interactúan, lo que se refleja en currículos poco flexibles, no pertinentes y modelos de enseñanza transmisionista que apelan a la memoria y la acumulación de datos. La investigación formativa surge como una manera de mejorar los procesos académicos, alineando docencia e investigación, para fomentar la adquisición de competencias investigativas, la generación de una cultura de investigación y el aprendizaje significativo. Este artículo presenta la caracterización del proceso de investigación formativa en el programa de ingeniería industrial de la I.U Pascual Bravo, mediante una metodología con alcance descriptivo y enfoque mixto, dividida en tres fases; como resultado se espera un mejoramiento en los procesos de formación e investigación de dicho programa.

Palabras claves: semillero de investigación, relación docencia-investigación, cultura investigativa.

\section{ABSTRACT}

The generation of a research culture and the integral formation of its professionals is a constant problem for the country's higher education institutions; the separation between academia and society isolates universities from the context in which they interact, which is reflected in inflexible, non-relevant curricula and transmissionist teaching models that appeal to memory and the accumulation of data. Formative research emerges as a way to improve academic processes, aligning teaching and research, to foster the acquisition of research competencies, the generation of a research culture, and meaningful learning. This article presents the characterization of the formative research process in the industrial engineering program of the I.U Pascual Bravo, by means of a methodology with descriptive scope and mixed approach, divided in three phases; as a result an improvement in the formation and research processes of said program is expected.

Key words: research seedbed, teaching-research relationship, research culture. 


\section{INTRODUCCIÓN}

La investigación formativa como estrategia pedagógica, se ha convertido en un elemento primordial para la generación de una cultura investigativa en las instituciones de educación superior (IES), implementada ya sea de forma transversal dentro de los contenidos curriculares y en las prácticas del docente en el aula a través de semilleros o por medio de la participación de estudiantes y docentes en proyectos de investigación (Ríos, Mesa y Zapata, 2010). De allí que tome relevancia la caracterización de este proceso en el Departamento de Producción de la I.U Pascual Bravo, de manera que se conozcan cuáles son sus fortalezas y debilidades, y se puedan elaborar planes de mejoramiento en este sentido (Maldonado, Landazábal, Hernandez, Ruiz y Vanegas, 2007).

En este artículo se realiza una caracterización del proceso de investigación formativa en el programa de ingeniería industrial, de modo que se pueda describir cómo el mismo ha impactado en la formación de profesionales en esta carrera y de qué manera ha servido para fomentar una cultura investigativa. Para tal efecto se planteó una metodología con alcance descriptivo y enfoque mixto, haciendo uso de la encuesta como instrumento para la obtención de información primaria, la cual se aplicó a docentes, estudiantes, empresarios y coordinadores de semilleros de investigación de la I.U Pascual Bravo. La información secundaria para la construcción del marco teórico se obtuvo de documentos desde diferentes bases de datos. Al final se muestran los resultados obtenidos con la aplicación del instrumento, incluidas tres herramientas de apoyo para la planeación estratégica cómo los árboles de problemas y de medios y fines, la Matriz DOFA y la Matriz de análisis de implicados. También se hace un análisis de los resultados obtenidos. Se espera que el artículo de investigación sirva como insumo para la elaboración de estrategias de mejoramiento en el proceso de investigación formativa en el programa, así como para futuras investigaciones en esta temática.

\section{INVESTIGACIÓN FORMATIVA}

El concepto de investigación formativa ha sido abordado por distintos autores, quienes han coincidido en algunos aspectos de su definición. Se trata de una estrategia de tipo pedagógico, con la que se busca dar solución a una problemática, con la mediación de un docente y la participación activa de estudiantes que se encargan de formular preguntas a partir de una situación inicial; la investigación formativa se enmarca dentro de la corriente pedagógica del constructivismo, desde el que se busca estimular el aprendizaje, fomentando la reflexión frente al proceso y la cultura investigativa, entendida como la generación de un espacio adecuado para la enseñanza y la práctica investigativa, así como las normas, valores y actitudes asociadas a esta (Ríos et al., 2010). Autores como Jiménez (2006), definen la investigación formativa como una manera de integrar currículo y práctica pedagógica con investigación, a través de las distintas estrategias posibles en un aula de clase; Maldonado et al. (2007) la analizan desde dos aspectos: el investigativo y el formativo, y cómo la misma está enfocada en el aprendizaje a lo largo de la vida, la generación de una cultura de investigación y de nuevo conocimiento que permita mejorar la comprensión del mundo.

Osorio (2008) a su vez, afirma que esta se apoya en los trabajos de grado como estrategia curricular, entendiendo los mismos como una manera de aplicar el conocimiento 
adquirido durante un proceso formativo. Parra (2004) opina que si la investigación formativa se trabaja de forma transversal a través del currículo, puede mejorar notablemente los procesos pedagógicos y didácticos, toda vez que ayuda a fortalecerlos a partir de la implementación del método científico en la resolución de problemas específicos. Rojas y Méndez (2013) mencionan algunos supuestos frente al tema como son el hecho de la poca experiencia investigativa de los estudiantes de pregrado, las inadecuadas metodologías de enseñanza, el manejo ineficiente de fuentes de información, currículos obsoletos y poco flexibles y falta de espacios extracurriculares para el fomento de la cultura investigativa. Otros autores como Rubio, Vila y Berlanga (2015) piensan que el estudiante debe ser el protagonista de su proceso de formación, y que los planes de estudio deben contener además de las competencias específicas del programa académico, competencias que apunten al fortalecimiento de su capacidad investigativa.

En este sentido, González (2006) ve en la investigación formativa una forma didáctica de solucionar problemas reales, teniendo en cuenta una formación pertinente que logre el desarrollo de las competencias requeridas por un entorno específico. Rojas (2009) habla de la misma como una manera de enseñar mediante el descubrimiento y la construcción, lo cual requiere flexibilidad y adaptabilidad en las estrategias pedagógicas.

Miyahira (2009) aporta a lo anterior cuando define la investigación formativa como una herramienta del proceso de enseñanza-aprendizaje, usada por un docente que tiene a su cargo sujetos en formación, quienes adquieren un conocimiento que será validado posteriormente en su vida profesional. Restrepo (2003) menciona la importancia de la relación entre docencia e investigación, al ser este un problema pedagógico y didáctico que aborda el papel de esta última en el proceso de aprendizaje; el mismo autor propone algunas formas para enseñar a investigar, tales como: realización de trabajos, elaboración de ensayos, proyectos con la participación de docentes y estudiantes, inclusión de asignaturas relativas a la investigación en los currículos y creación de semilleros de investigación. En este misma línea, Silva, Torres, González y Sarmiento (2008) mencionan algunas maneras de integrar docencia e investigación, tales como la inclusión de asignaturas relativas a la investigación en los planes de estudio, el establecimiento de vínculos entre docentes y estudiantes a través de proyectos y espacios extracurriculares, y por último potenciar las competencias investigativas mediante los semilleros de investigación.

Peláez y Montoya (2013) hablan de la investigación formativa como la capacidad para emplear los métodos de investigación en los procesos de enseñanza, con el fin de desarrollar en los estudiantes competencias de tipo investigativo y mejorar la relación docencia-investigación. Bolívar (2013) por su parte, hace hincapié en la diferencia entre formación investigativa e investigación formativa, dado que el fin de esta última debe ser el de la generación de una cultura de investigación. De igual manera, centra su análisis en el problema de la relación entre docencia e investigación y la forma como se está enseñando a investigar.

Jiménez (2006) también hace la diferenciación entre investigación en sentido estricto e investigación formativa, aclarando que en la primera se desarrollan de proyectos formales y con líneas definidas, dentro de grupos de investigación en los que participan generalmente los docentes como investigadores principales y cuyo objetivo es la generación de nuevo conocimiento en una disciplina específica. Ríos et al. (2010) coinciden con los autores anteriores cuando afirman que esta se centra en el proceso pedagógico, de enseñanza de la investigación y generación de una cultura investigativa. Otros autores como Espinoza (2016) 
hablan de la investigación formativa como una manera de formar en investigación a través de la participación de estudiantes en proyectos que logren familiarizarlos con el método científico. En este sentido, Vila, Rubio y Berlanga (2014) mencionan la importancia del aprendizaje basado en problemas, dado que permite que los estudiantes pongan en práctica las distintas competencias adquiridas en sus carreras.

\section{APRENDIZAJE, DOCENCIA E INVESTIGACIÓN}

El análisis de la relación entre métodos de enseñanza, práctica docente, planeación curricular, contexto de aplicación e investigación es fundamental para hacer del aprendizaje algo significativo; Trujillo (2007) por ejemplo, habla de la relación entre academia y cotidianidad, dándole importancia a la oferta de contenidos académicos acordes con la realidad en la que se desenvuelven los sujetos, ya que debido a estructuras curriculares no pertinentes y poco flexibles, se da una separación entre ambas. Berrouet (2008) analiza el problema del aprendizaje repetitivo y de corte transmisionista presente en muchas universidades, donde el estudiante asimila conceptos de forma pasiva, usando básicamente la memoria y aplicando fórmulas, sin entender la complejidad de su proceso formativo y de las relaciones que se pueden establecer entre las distintas variables que lo condicionan. De igual manera, Ossa y Sierra (2001) hablan de una limitada y reduccionista, la que se fundamenta en un aprendizaje repetitivo y mecanicista.

Velásquez (2008) menciona la importancia de la relación comunidad-universidadempresa, ya que el conocimiento generado en las instituciones educativas debe ser pertinente y responder a las necesidades de formación de una sociedad específica, así como los requerimientos de capital humano del sector productivo, de manera que se aporte al desarrollo económico y se solucionen las problemáticas relativas al entorno; más aún en un mundo globalizado en dónde la competitividad es muy grande y se requiere que las regiones sean capaces de afrontar los retos que impone una economía de mercado (Rojas, Cardona y Gallego, 2017). Así mismo, opina que la práctica docente y la investigación deben enfocarse hacia una actitud reflexiva frente al conocimiento y los procesos de formación, cambiando el rol tradicional del docente transmisionista por un docente-investigador que pueda fomentar el trabajo en equipo, la co-creación y la creatividad a partir de la solución de problemas usando el método científico, lo que permite mejorar la capacidad investigativa de la comunidad a partir de la formación adecuada del capital humano.

Según Osorio (2008), un problema fundamental en la investigación es el uso de métodos de enseñanza obsoletos, con estrategias poco innovadoras; además, esta se ve afectada por la baja calidad de la educación, la poca cobertura, el déficit de capital humano calificado, los problemas en lectura y escritura tanto de docentes como estudiantes, la poca flexibilidad e interdisciplinariedad de los currículos, la separación entre docencia e investigación y la carencia de comunidades científicas y académicas consolidadas. Rojas y Méndez (2013) hablan de la poca correlación entre la practica docente y la formación científica, lo que está presente no solo en las universidades, las cuales están enfocadas en la profesionalización, sino también en las instituciones de educación básica y media, donde no se brinda una formación con un mínimo rigor científico; los autores concluyen que debe darse un cambio en la práctica docente, direccionándola hacia la promoción de la cultura investigativa desde las prácticas pedagógicas, y haciendo del aprendizaje algo significativo y pertinente. 
Parra (2004) resalta la importancia de la articulación entre docencia e investigación, lo que, según el autor, trae múltiples beneficios tales como: mejoramiento en el desarrollo de los contenidos curriculares, mayor comprensión del proceso de generación de conocimiento, motivación hacia el aprendizaje autónomo, configuración de una estructura mental ordenada, desarrollo del pensamiento crítico, discursivo y holístico, capacidad para formulación de problemas y planteamiento de soluciones, además, permite situar al docente en un rol de facilitador en el proceso de formación. Berrouet (2007) menciona algunas problemáticas relativas a los procesos de formación en instituciones de educación superior, tales como: ausencia de comunidades académicas, desarticulación entre investigación y contenidos curriculares, poco rigor académico, modelos de enseñanza transmisionistas, falta de pensamiento crítico frente a la labor docente, desarticulación con la realidad política, económica, social e histórica del país.

\section{SEMILLEROS DE INVESTIGACIÓN E INVESTIGACIÓN FORMATIVA}

El semillero de investigación se entiende como un espacio de formación, en el que se puede complementar la práctica pedagógica y el currículo con elementos de investigación en el aula, a partir de la formulación de preguntas relativas al entorno y el análisis de las posibles alternativas de solución (Ossa y Sierra, 2001). La finalidad de los semilleros de investigación debe ser el desarrollo de la capacidad investigativa a través de la interacción entre estudiantes y profesores, lo que favorece el mejoramiento académico a la vez que se busca un impacto positivo en la comunidad, favoreciendo la formación integral al permitir superar los esquemas rígidos de enseñanza de carácter sumatorio y memorísticos que impiden la profundización de contenidos. Lo anterior redunda en la generación de una cultura investigativa en las instituciones. Maldonado et al. (2007) ven los semilleros de investigación como comunidades de aprendizaje, en donde se buscar dar respuesta a preguntas surgidas a través del autoaprendizaje y la práctica investigativa. A su vez, Molineros (2009) habla de dos tendencias presentes en los semilleros: la primera es aquella donde el estudiante va desarrollando una idea de investigación propia, acompañado de un tutor; la segunda se presenta cuando un docente experto quien lidera la investigación y el estudiante acompaña dicho proceso.

Frente al tema, González (2008) afirma que el semillero de investigación sirve para fomentar la cultura investigativa, al brindar herramientas para el desarrollo de competencias investigativas que le permita a estudiantes y docentes acercarse a la realidad social de manera que puedan formular proyectos como alternativas de solución a las problemáticas de la comunidad; de esta manera, se convierte en una estrategia para el fomento de la investigación formativa y el mejoramiento de la relación entre academia y sociedad. De igual manera, se fortalece la capacidad investigativa y se mejora la calidad académica; además, se motiva al trabajo en grupos y en redes. Lammas (2006) define los semilleros de investigación como el espacio adecuado para cultivar futuros investigadores, que sean capaces de dar respuestas a los problemas complejos de la sociedad, a partir de la continua reflexión para la construcción de conocimiento; así mismo menciona la importancia de articular el trabajo investigativo con los procesos formativos. Rendón (2002) define el semillero de investigación como un espacio en el que se conjugan estrategias dentro del proceso formativo, para fomentar la cultura investigativa. 
Trujillo (2007) menciona la importancia de los semilleros de investigación como una estrategia coherente y pertinente para generar una cultura investigativa y mejorar los procesos de formación en investigación. Frente al mismo tema, Berrouet (2008) habla de la manera como los semilleros de investigación pueden propiciar el trabajo en equipo y comprensión de la realidad desde una visión compleja y desde otras perspectivas de análisis, asumiendo a su vez el proceso de formación cómo permanente descubrimiento.

Ríos (2009) entiende los semilleros de investigación una estrategia para que los estudiantes se integren a la formación investigativa, atendiendo una necesidad de formación en competencias científicas. Molina, Martinez, Marín y Vallejo (2012) resaltan el aporte de los semilleros de investigación a la autonomía en el aprendizaje y la adquisición de competencias investigativas necesarias en todo profesional, las cuales también mencionan Landazábal, Pineda, Páez, Téllez y Ortiz (2010), quienes las definen como una mezcla de conocimientos, habilidades, actitudes y aptitudes.

\section{METODOLOGÍA}

La presente investigación tiene un alcance descriptivo con enfoque mixto (Hernández, Collao \& Baptista, 2014) y está dividida en 3 fases. En la primera se obtuvo información bibliográfica sobre la temática relativa al proyecto y se formuló el mismo. En la segunda se procedió a elaborar el instrumento para la recopilación de información primaria, el que se enfocó en 4 grupos específicos (encuesta dirigida a estudiantes, docentes, semilleristas y empresarios); de igual manera se llevó a cabo la aplicación, sistematización y análisis de resultados. En la tercera fase, y con base en los resultados obtenidos en la fase anterior, se procedió a elaborar los instrumentos de análisis estratégico que servirán como insumo para la elaboración de planes de mejoramiento dentro del programa de Ingeniería Industrial de la I.U Pascual Bravo, como son: Matriz DOFA, Árboles de Problemas y de Medios y Fines, Matriz de Análisis de Implicados. Las etapas del estudio fueron:

\section{Fase 1:}

- Recolección de información bibliográfica para formulación del proyecto.

- Análisis de información secundaria obtenida.

- Formulación del proyecto

Fase 2:

- Elaboración de instrumento de obtención de información primaria.

Validación de instrumento y realización de prueba piloto.

- Aplicación de encuestas.

- Análisis de información obtenida.

Fase 3:

- $\quad$ Elaboración arboles de problemas y de medios y fines.

- Construcción de matriz DOFA.

- Construcción de Matriz de análisis de implicados.

- Elaboración de documento final, con resultados y conclusiones. 


\subsection{ENCUESTAS}

La encuesta diseñada se aplicó a 4 grupos específicos: integrantes del semillero de investigación SEPROCA de la Institución Universitaria Pascual Bravo en los últimos 4 semestres (31), coordinadores de semilleros de investigación de la facultad de producción y diseño (4), docentes (25) y empresarios (16); esta fue enviada vía correo electrónico para ser diligenciada, y contenía preguntas relativas a aspectos tales como: infraestructura y estrategias de investigación en el programa, nivel académico, nivel de formación de docentes, adquisición de competencias investigativas y desempeño de los practicantes en las empresas. Adicionalmente, en el comité curricular del Departamento de Producción se construyó una matriz de evaluación de los anteproyectos y proyectos de grado de los estudiantes, y se tomó información brindada por la oficina de prácticas de la institución. La información obtenida se organizó en tablas, gráficos y hojas de Excel para su posterior análisis.

\section{RESULTADOS}

La tabla 1 muestra los resultados obtenidos mediante la aplicación del instrumento a los 4 grupos de interés, tomando como referencia las preguntas más relevantes para este estudio:

Tabla 1. Resultados encuesta

\begin{tabular}{|c|c|}
\hline \multicolumn{2}{|r|}{ Estudiantes semilleros } \\
\hline Tiempo de permanencia en el semillero & $\begin{array}{l}\text { 45.2\%: } 1 \text { semestre; } 22.6 \%: 50 \text { mas semestres; } 9.7 \%: 3 \\
\text { semestres, } 9.7 \%: 2 \text { semestres; } 6.5 \%: 4 \text { semestres y el } \\
3.2 \% \text { : recien ingresados }\end{array}$ \\
\hline ¿Trabaja actualmente? & $\begin{array}{l}\text { El } 67.7 \% \text { de los integrantes del semillero trabajan } \\
\text { actualmente }\end{array}$ \\
\hline ¿Qué lo motivo a ingresar al semillero? & $\begin{array}{l}\text { 45.2\%: ampliar conocimientos; } 35.5 \% \text { : aprender sobre } \\
\text { investigación; } 12.9 \% \text { : como opción de trabajo de grado ; } \\
\text { 6.5\%: acceso a descuento en matricula }\end{array}$ \\
\hline $\begin{array}{l}\text { ¿Qué expectativas tiene frente al } \\
\text { semillero? }\end{array}$ & $\begin{array}{l}\text { 80.6\% participación en proyectos; } 61.3 \% \text { vinculación al } \\
\text { grupo de investigación; } 54.8 \% \text { : asistencia a eventos; } \\
54.8 \% \text { : certificación de experiencia; } 51.6 \% \text { : realización } \\
\text { de ponencias ; } 22.6 \% \text { : producción científica. }\end{array}$ \\
\hline $\begin{array}{l}\text { ¿Los docentes de las distintas } \\
\text { asignaturas motivan la investigación? }\end{array}$ & $\begin{array}{l}\text { El } 70.6 \% \text { opinan que los docentes si motivan la } \\
\text { investigación en el aula. }\end{array}$ \\
\hline $\begin{array}{l}\text { ¿Considera que se usan estrategias } \\
\text { adecuadas de enseñanza-aprendizaje en } \\
\text { el aula? }\end{array}$ & $\begin{array}{l}\text { El } 70 \% \text { de los estudiantes opinan que las estrategias que } \\
\text { usan los docentes son adecuadas, el } 18.9 \% \text { consideran que } \\
\text { no son y el } 11.1 \% \text { opina que todo depende del docente a } \\
\text { cargo. }\end{array}$ \\
\hline $\begin{array}{l}\text { ¿Considera que los proyectos de aula de } \\
\text { las distintas asignaturas han contribuido } \\
\text { a la formación en competencias } \\
\text { investigativas? }\end{array}$ & $\begin{array}{l}\text { El } 75,3 \% \text { de los encuestados respondieron que los } \\
\text { proyectos de aula sí han contribuido a formación de } \\
\text { competencias investigativas. }\end{array}$ \\
\hline
\end{tabular}




\begin{abstract}
¿Qué competencias considera que ha Responsabilidad, redacción, análisis, trabajo en equipo, adquirido gracias a su participación en expresión oral, confianza, capacidad de solución de el semillero y que le son útiles en su problemas, uso de bases de datos, comprensión lectora, labor profesional? disciplina, puntualidad y compromiso.
\end{abstract}

\section{Coordinadores semilleros}

¿Qué tipo de metodología emplea en los procesos de formación dentro del $\mathrm{ABP}$, marco lógico, método cientifico, trabajo en equipo e semillero?

¿Qué tipo de producción científica y académica tiene el semillero?

¿Cuáles son las líneas de investigación del semillero? investigación aplicada.

Innovaciones generadas en la gestión empresarial, ponencias, proyectos de investigación, talleres de creación, en proceso: cartillas y manuales

Logística, producción , calidad, diseño imagen y sociedad tic, multimedia e innovación en materiales y diseño sostenible.

¿En que eventos académicos ha participado el semillero?

Redcolsi, Simposio de Productividad, Simposio Internacional de Diseño Sostenible, Encuentro de Semillero de Investigación ACIET.

\section{Docentes}

¿Utiliza algún tipo de metodología para la presentación de trabajos de sus estudiantes?

¿Qué propuesta metodológica implementa en los proyectos de aula de las asignaturas a cargo?

Exposiciónes, cuestionarios, equipos colaborativos, discusión en grupos, visitas empresariales, ensayos, reporte, , artículos, videos descriptivos, resolución de problemas y estudios de caso, pruebas específicas .

Con base a la teoría vista en clase, los estudiantes deben realizar un trabajo de campo, donde se evidencie trabajo de investigación y socialicen a sus compañeros las propuestas de solución de problemáticas reales.

En general los docentes tienen experiencia en investigación

¿Tiene experiencia en investigación validada por Colciencias, algunos productos son: artículos (producción validada por Colciencias)? de revista, libro, software, innovación en producción empresarial, ponencias y posters

¿De qué manera fomenta la cultura investigativa en sus asignaturas?

Consultas, lectura de artículos científicos, investigación en bases de datos, desarrollo de proyectos de aula, , uso del lenguaje técnico.

\section{Empresarios}

Área o departamento en el que se desempeña el practicante o egresado

El $76.9 \%$ de los practicantes o egresados se desempeñan en el área de producción, el 7,7\% en el área de mercadeo y publicidad, $7.7 \%$ administrativa y $7,7 \%$ en otras.

El 53,8\% de los empresarios respondieron que su nivel de Nivel de satisfacción con el desempeño satisfacción con el desempeño de los practicantes o del practicante o egresado egresados es alta, el 38,5\% respondió que su nivel de satisfacción es muy alto y el $7,7 \%$ es medio.

Capacidad de creatividad e innovación para el desarrollo Razones principales para vincular de oportunidades de mejora de la empresa: 46,2\%; graduados o practicantes de la I.U Pascual Bravo
Capacidad para identificar, planear y resolver problemas: 38,5\%; Perfil ocupacional multiple: 7,7\%; Capacidad de formular y ejecutar proyectos: $7,7 \%$ 


\begin{tabular}{|l|l|}
\hline $\begin{array}{l}\text { Competencias específicas mas } \\
\text { importantes de un egresados o } \\
\text { practicante de la institución }\end{array}$ & $\begin{array}{l}\text { Utilización adecuada del tiempo, trabajo en equipo, } \\
\text { valores éticos y profesionales, identificar, planear y } \\
\text { resolver problemas, generar y desarrollar ideas creativas } \\
\text { para el desarrollo de oportunidades de mejora, abstracción, } \\
\text { análisis y síntesis, adaptación al cambio, trabajo bajo } \\
\text { presión, expresión oral, uso de herramientas informáticas, } \\
\text { toma de decisiones, manejo de segundo idioma }\end{array}$ \\
\hline $\begin{array}{l}\text { Evaluación del nivel de logro de } \\
\begin{array}{l}\text { competencias por parte de practicantes } \\
\text { y egresados de la institución }\end{array}\end{array}$ & $\begin{array}{l}\text { La mayor parte de las competencias especificas tuvieron } \\
\text { una valoración alta por parte de los empresarios. }\end{array}$ \\
\hline
\end{tabular}

Fuente. Elaboración propia.

La encuesta completa arrojó los siguientes resultados: El rango de edades de los estudiantes de ingeniería industrial está entre 21 a 30 años en un mayor porcentaje $(72,1$ $\%)$, lo cual se explica en el hecho que la mayoría de ellos trabajan (77,8 \% están en jornada nocturna). Muchos de ellos no participan en ningún semillero de investigación o no los conocen, sin embargo les gustaría pertenecer a alguno (43,5\%), pero presentan problemas con el tiempo dadas sus ocupaciones laborales. El 55,6 \% de los encuestados considera que la institución cuenta con una infraestructura adecuada para la investigación; así mismo, el $58,3 \%$ opinan que los docentes motivan la investigación dentro del aula. Un $75 \%$ califican el nivel académico del programa como bueno o excelente y un 55,6\% piensan que se usan estrategias adecuadas de enseñanza-aprendizaje en las clases.

El 91,7 \% de los estudiantes piensan que es importante la investigación formativa dentro del programa académico y un 58,3\% afirman que los proyectos de aula si contribuyen a la formación en competencias investigativas, pero se deben articular en todas las asignaturas. Por otro lado, el $68 \%$ de los docentes afirman emplear algún tipo de metodología para la presentación de trabajos y proyectos de aula, diferente a la clase magistral, entre las que se incluyen la guía institucional para proyectos de investigación, el análisis de casos y la aplicación de conceptos para la solución de un problema en una empresa especifica.

El $88 \%$ de los docentes tienen formación de posgrado (maestría y/o especialización) y la mayoría tiene 4 años o más de experiencia docente $(60 \%)$. El $40 \%$ de los docentes pertenece a algún grupo de investigación y el $35 \%$ afirman tener algún tipo de producción investigativa. El $58 \%$ no han participado en ningún tipo de proyecto con algún grupo o semillero de investigación de la institución. La mayoría de los docentes opinan que se motiva la cultura investigativa en su asignatura ( $83 \%$ ), a través del uso de distintas herramientas.

La mayoría de los integrantes del semillero de investigación SEPROCA están entre el 4to y 10mo semestre de la carrera, y un 54,8 \% llevan más de un semestre en este; el 67,7 $\%$ tienen edades entre los 16 y los 25 años y el 54,8 \% pertenecen a la jornada diurna; el $67,7 \%$ trabaja actualmente. Dentro de los motivos que los llevaron a ingresar el semillero están: adquirir conocimiento, aprender temas de investigación y la selección de modalidad de trabajo de grado. El 80,6\% tienen como expectativa en el grupo la participación en proyectos de investigación, el $54.8 \%$ la vinculación a un grupo de investigación, y un 54.8\% asistencia a eventos académicos. El $71 \%$ de los semilleristas piensan que la institución cuenta con una infraestructura adecuada para la investigación y el $80.6 \%$ opinan que los docentes motivan la investigación en el aula. El $96 \%$ de los semilleristas piensan que el nivel del programa académico 
es bueno o excelente y el $83,9 \%$ que se usan estrategia adecuadas de enseñanza-aprendizaje. El 93,5\% considera que pertenecer al semillero de investigación les ha contribuido en su proceso de formación y que la investigación formativa es muy importante en el programa.

El $100 \%$ de los semilleristas ha participado en algún proyecto de investigación. Entre las herramientas metodológicas que han mejorado gracias a la participación en el semillero se encuentran: manejo de fuentes de información, análisis cualitativo, nivel de redacción y análisis matemático. De igual manera, han adquirido competencias tales como: redacción y análisis, aplicación del conocimiento en la práctica, trabajo en equipo, liderazgo, responsabilidad, interpretación de información. El 90,3\% de los semilleristas consideran importantes los proyectos de aula, ya que en ellos se adquieren competencias investigativas y un 96,8 opinan que han tenido un buen acompañamiento por parte del coordinador del semillero.

El $75 \%$ de los coordinadores de semilleros de investigación de la facultad emplean alguna metodología en los procesos de investigación formativa; la producción investigativa de dichos semilleros se centra en: procesos de intervención en empresas, realización de ponencias, talleres de creación, realización de cartillas o manuales. Cada semillero tiene su línea de investigación establecida, la de SEPROCA es productividad y calidad. Los semilleros participan cada año en los eventos que programa la red colombiana de Semilleros de Investigación RedCOLSI, además SEPROCA participa en la Red de Productividad REDPROD, como apoyo logístico en el simposio anual de productividad y con la presentación de ponencias y posters en el mismo; dicho semillero está adscrito al grupo de investigación QUALIPRO. Dentro de los retos que se plantean en el semillero están los de participar en eventos nacionales e internacionales, así como la publicación de artículos académicos.

El $50 \%$ de las empresas encuestadas son medianas, el 25\% grande, y las demás son pequeña y microempresas, el 83,3\% pertenecen al sector privado. El área donde más se desempeñan practicantes o egresados en estas empresas es la de producción (75\%); un $58,3 \%$ de los empresarios manifiestan un alto nivel de satisfacción con el desempeño de los practicantes o egresados de la institución y un $33.3 \%$ muy alto; las razones más comunes por las cuales estos son contratados son: capacidad de creatividad, innovación y desarrollo de oportunidades, capacidad para identificar, planear, verificar, controlar y mejorar procesos productivos. Las empresas le dan una calificación alta en la valoración de las siguientes competencias específicas: trabajo en equipo, logros de objetivos, propuesta de mejoramiento, competencias actitudinales, utilizan adecuado el tiempo, trabajo independiente sin supervisión, aplican valores éticos y profesionales, buscan y analizan para dar solución, adaptación al cambio, trabajo bajo presión.

Tomando como referencia la información suministrada por la oficina de prácticas de la institución, se tiene que para el semestre 2018-1, de un total de 34 practicantes que participaron en la encuesta de evaluación del proceso de practica ( 7 de ingeniería industrial y 27 de producción industrial), la totalidad opina que el programa es pertinente, debido entre otras cosas a: contenido y aplicabilidad de las asignaturas, enfoque de los programas hacia la industria, nivel de exigencia académico y aprendizaje significativo.

El análisis de la matriz de valoración de anteproyectos y proyectos de grado elaborado por el comité curricular del Departamento de Producción en el semestre 2018-1, muestra los siguientes resultados: un 34,7 \% de los anteproyectos son aprobados sin modificaciones, y se tiene una nota promedio de valoración de 4.0 (en una escala de 0 a 5); los proyectos que son aprobados con modificaciones $(65,3 \%)$ presentan falencias en aspectos como la definición del problema de investigación, formulación de objetivos, metodología mal 
definida, redacción en general, manejo de normas de citación, poca profundidad del marco teórico y cronogramas de trabajo mal diseñados.

En cuanto a los proyectos de grado terminados, se tiene una nota promedio de 4,3. Dichos proyectos fueron evaluados en estos aspectos: planteamiento del problema, antecedentes, objetivos, justificación, marco teórico, metodología, resultados esperados, cumplimiento de objetivos, conclusiones y presentación. Las observaciones generales que se hicieron a los trabajos tienen que ver con aspectos relativos a la redacción, el análisis de los resultados obtenidos y su validación, el uso de referencias y la descripción de la metodología a emplear. Se hizo además un análisis de la coherencia entre la valoración del anteproyecto y la del proyecto de grado terminado, dando como resultado una coherencia promedio del $88 \%$ entre ambas valoraciones.

\section{1. ÁRBOLES DE PROBLEMAS Y DE MEDIOS Y FINES, MATRIZ DOFA, MATRIZ DE ANÁLISIS DE IMPLICADOS}

Con base en la información obtenida (primaria y secundaria), se procedió a elaborar el árbol de problemas y el de medios y fines referentes a la temática especifica de investigación formativa en el programa de ingeniería industrial de la I.U Pascual Bravo, tal como se presentan en las figuras 1 y 2, así como la matriz DOFA mostrada en la tabla 1, y la Matriz de Análisis de Implicado (Importancia vs Dominio), correspondiente a la figura 3. Es preciso anotar que dicha información se complementó con información obtenida desde la jefatura de departamento y la decanatura de dicho programa.

Figura 1. Árbol de problemas investigación formativa



Fuente. Elaboración propia. 
Figura 2. Árbol de medios y fines investigación formativa

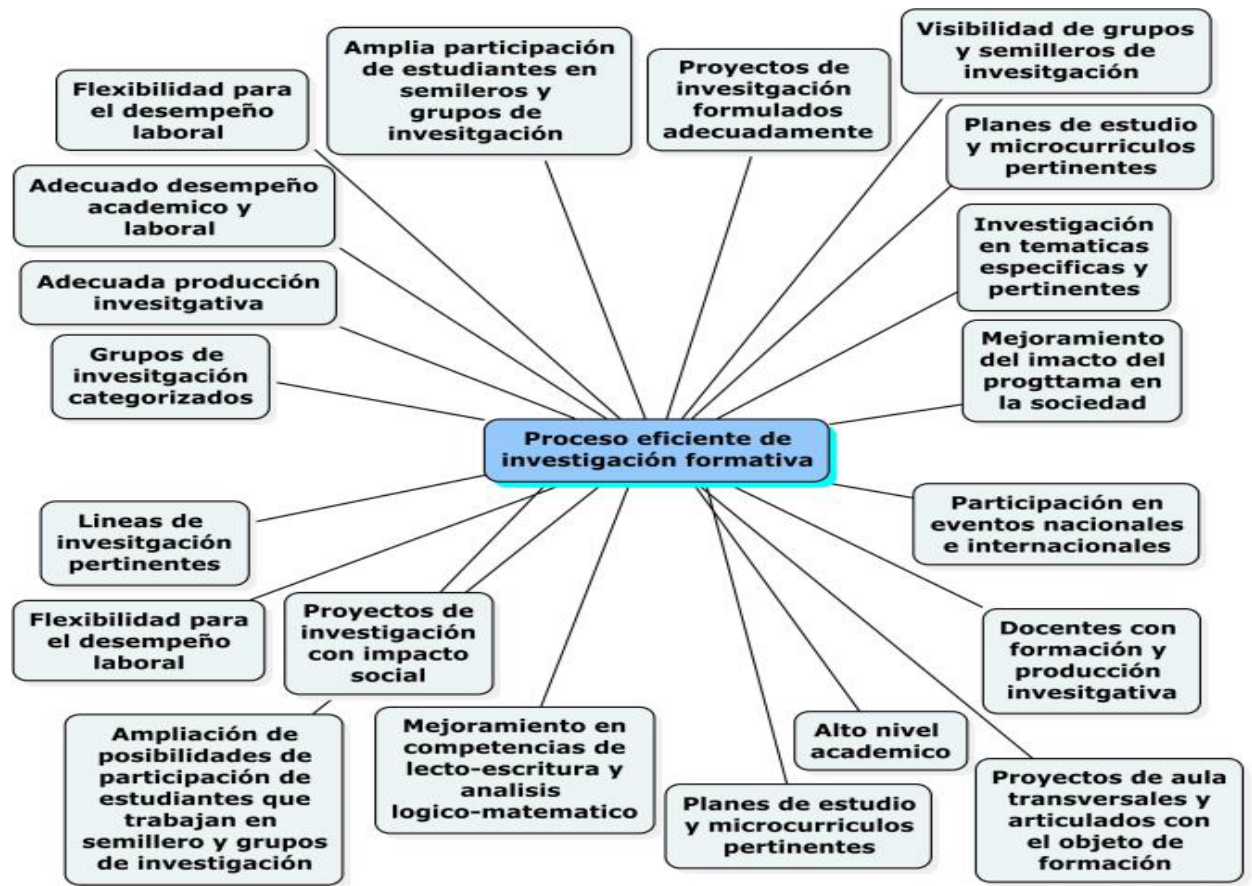

Fuente. Elaboración propia.

\section{Tabla 2. Matriz DOFA}

\begin{tabular}{|c|c|c|}
\hline \multicolumn{3}{|c|}{ MATRIZ DOFA } \\
\hline $\begin{array}{l}\text { Objetivo } \\
\text { Mejorar los procesos } \\
\text { relacionados con la investigación } \\
\text { formativa en el programa de } \\
\text { Ingenieria Industrial de la I.U } \\
\text { Pascual Bravo }\end{array}$ & \begin{tabular}{|l|} 
Fortalezas \\
Relación con la industria. \\
Aceptación del programa. \\
Semillero de investigación. \\
Infraestructura para investigación. \\
Proyectos de aula. \\
Modelo de formación por \\
competencias. \\
Pertenencia a redes. \\
\end{tabular} & $\begin{array}{l}\text { Debilidades } \\
\text { Competencias lógico-matemáticas } \\
\text { y de lecto-escritura. } \\
\text { Desarticulación de planes de } \\
\text { estudio con el contexto y poca } \\
\text { flexibilidad. } \\
\text { Formación de docentes en } \\
\text { investigación. } \\
\text { Población estudiantil trabajadora. }\end{array}$ \\
\hline $\begin{array}{l}\text { Oportunidades } \\
\text { Llegada de la industria } 4.0 . \\
\text { Aumento de empresas. } \\
\text { Convenios de carácter nacional e } \\
\text { internacional. } \\
\text { Necesidades de la región y del } \\
\text { país en temas de investigación. } \\
\text { Eventos de investigación. }\end{array}$ & $\begin{array}{l}\text { Potencialidades } \\
\text { Inversión en constante } \\
\text { infraestructura y aumento del } \\
\text { presupuesto en investigación. } \\
\text { Contratación de nuevos docentes. } \\
\text { Sinergia con ITM y Colmayor. } \\
\text { Apertura Convocatorias para } \\
\text { proyectos de investigación. }\end{array}$ & $\begin{array}{l}\text { Desafíos } \\
\text { Industria 4.0. } \\
\text { Nuevas generaciones de } \\
\text { estudiantes. } \\
\text { Planes de desarrollo local, } \\
\text { departamental y nacional. } \\
\text { Nuevos paradigmas educativos en } \\
\text { la formación por competencias. } \\
\text { Globalización de la economía. }\end{array}$ \\
\hline
\end{tabular}




\begin{tabular}{|c|c|c|}
\hline $\begin{array}{l}\text { Amenazas } \\
\text { Llegada de nuevas IES con } \\
\text { programas similares. } \\
\text { Crisis de la industria. } \\
\text { Disminución del presupuesto } \\
\text { para investigación por parte de la } \\
\text { nación. } \\
\text { Poca adaptabilidad a los nuevos } \\
\text { modelos de enseñanza. } \\
\text { Deserción estudiantil }\end{array}$ & $\begin{array}{l}\text { Riesgos } \\
\text { Inversión inadecuada en } \\
\text { infraestructura. } \\
\text { Errores en contratación docente. } \\
\text { Líneas de investigación mal } \\
\text { formuladas. } \\
\text { No continuidad de las políticas en } \\
\text { investigación. }\end{array}$ & $\begin{array}{l}\text { Limitaciones } \\
\text { Prepuesto. } \\
\text { Espacio físico. } \\
\text { Legales por ser I.U. }\end{array}$ \\
\hline
\end{tabular}

Fuente. Elaboración propia.

Figura 3. Resultados valoración de análisis de implicados

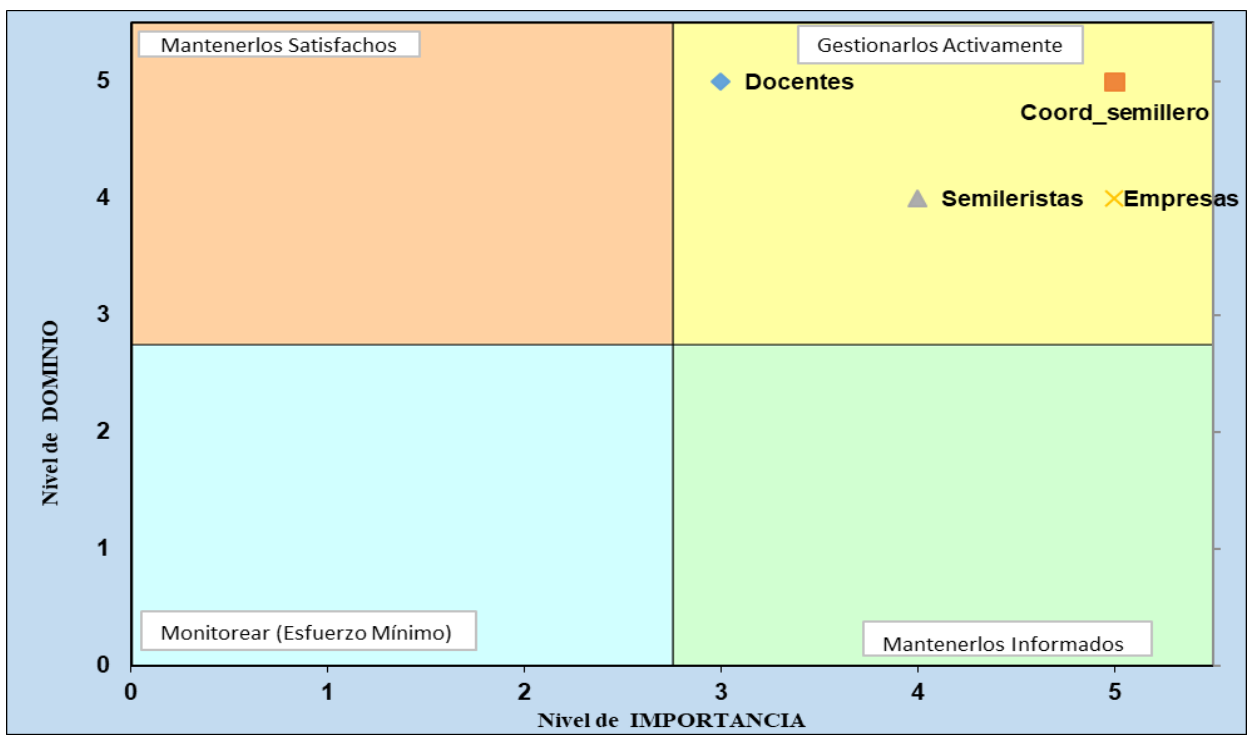

Fuente. Elaboración propia. 
Tabla 3. Matriz de análisis de implicados

\begin{tabular}{|c|c|c|c|c|}
\hline Involucrados & Responsabilidad & Expectativas & Actitud & Acciones \\
\hline Docentes & Formadores & $\begin{array}{l}\text { Proceso exitoso } \\
\text { de formación } \\
\text { integral }\end{array}$ & Partidario & $\begin{array}{l}\text { Formación continua en in } \\
\text { vestigación, mejoras salaria- } \\
\text { les, incentivos por participa- } \\
\text { ción en proyectos de investi- } \\
\text { gación; concientización fren- } \\
\text { te a la importancia de la in- } \\
\text { vestigación formativa }\end{array}$ \\
\hline Coord_semillero & $\begin{array}{l}\text { Gestión } \\
\text { semilleros }\end{array}$ & $\begin{array}{l}\text { Formación en } \\
\text { competencias } \\
\text { investigativas a } \\
\text { semilleristas; } \\
\text { desarrollo de } \\
\text { productos de } \\
\text { investigación } \\
\text { formativa }\end{array}$ & Líder & $\begin{array}{l}\text { Apoyo para participación en } \\
\text { eventos, mejoras salariales, } \\
\text { incentivos por proyectos de } \\
\text { investigación presentados, } \\
\text { formación continua }\end{array}$ \\
\hline Semilleristas & $\begin{array}{l}\text { Participación en } \\
\text { proyectos }\end{array}$ & $\begin{array}{l}\text { Adquisición de } \\
\text { competencias en } \\
\text { investigación }\end{array}$ & Partidario & $\begin{array}{l}\text { Incentivos económicos según } \\
\text { proyectos, posibilidad de } \\
\text { continuar proyectos de inves- } \\
\text { tigación como egresados, } \\
\text { viáticos para asistencia } \\
\text { eventos nacionales e interna- } \\
\text { cionales, formación continua }\end{array}$ \\
\hline Empresas & $\begin{array}{l}\text { Evaluadores } \\
\text { competencias }\end{array}$ & $\begin{array}{l}\text { Ingenieros con } \\
\text { formación } \\
\text { ajustada a } \\
\text { perfiles } \\
\text { requeridos } \\
\end{array}$ & Partidario & $\begin{array}{l}\text { Generar espacios de dialogo } \\
\text { con los empresarios para la } \\
\text { construcción de planes de es } \\
\text { tudio y estrategias en temas } \\
\text { relativos a la investigación }\end{array}$ \\
\hline
\end{tabular}

Fuente. Elaboración propia.

\subsection{ANÁLISIS DE RESULTADOS}

La percepción del estudiante frente al proceso de investigación formativa en la institución es positiva, aunque aún no es totalmente satisfactoria. En general, resaltan el hecho que se estén haciendo esfuerzos en investigación, así como las estrategias de enseñanzaaprendizaje empleadas por los docentes en el aula; sumado al hecho que en casi todas las asignaturas se estén implementando los proyectos de aula como una manera de aplicar los conocimientos adquiridos en el proceso de formación, lo cual a su vez fomenta la cultura investigativa y ayuda en el mejoramiento del nivel académico. Es de anotar que un alto porcentaje de los estudiantes de la I.U Pascual Bravo trabajan, por lo cual no tienen mucho tiempo para participar en los semilleros y grupos de investigación, los que están constituidos mayoritariamente por estudiantes de la jornada diurna.

Los docentes han asimilado de buena manera la estrategia de los proyectos de aula como una manera de motivar la investigación, pero ven una oportunidad de mejora en la integración de estos a través de todo el currículo, de manera que tenga un mayor impacto en 
el proceso formativo; se tiene un alto porcentaje de docentes con formación de posgrado, pero aun la producción científica no es muy alta, y está soportada en su mayoría por los trabajos de grado, asesorías y consultorías.

Los integrantes del semillero de investigación SEPROCA, adscrito al programa de ingeniería industrial, valoran de forma positiva su participación en este; opinan que les ha servido para la generación de competencias investigativas y laborales que les permiten un mejor desempeño en las organizaciones. Dado que el enfoque de este semillero son las empresas, a partir de intervenciones en las mismas en temas de productividad y calidad, los resultados obtenidos han servido para acercar la institución a la industria local, con la cual se ha tenido históricamente muy buena relación; además, esto de hacer del aprendizaje algo significativo e impactar de forma positiva a la sociedad. Asimismo, los semilleristas opinan que la calidad del programa académico de ingeniería industrial es muy buena y valoran los esfuerzos institucionales por mejorar en los procesos de investigación.

Los coordinadores de semilleros de investigación de la Facultad de Producción y Diseño de la I.U Pascual Bravo en su totalidad utilizan algún tipo de estrategia metodológica para desarrollar los proyectos de investigación dentro del grupo. Si bien los intereses de cada uno de ellos en materia investigativa son distintos, se tiene producción científica y se ha logrado visibilidad a partir de la participación en eventos y redes de conocimiento; se tiene como propuesta de mejora el desarrollo de proyectos de investigación más rigurosos y que generen una mayor producción académica, sin dejar de lado el enfoque formativo de los semilleros.

Los resultados arrojados en la encuesta realizada en las empresas permiten deducir que se tiene una impresión positiva de los practicantes y egresados del programa de ingeniería industrial; resaltan que estos tienen competencias personales y laborales que les permiten desempeñarse de forma eficiente en las organizaciones, y que su principal área de actuación es la parte productiva. Sin embargo, se deben fortalecer en otros aspectos también relativos a la carrera, como las finanzas, gerencia y análisis matemático complejo. Tanto las empresas como los practicantes y egresados ven muy pertinente el programa académico, y valoran el enfoque que se da desde los contenidos curriculares hacia la aplicabilidad del conocimiento en contextos reales y la capacidad para solucionar problemas específicos en las empresas. Se ve como una oportunidad de mejora fortalecer el área de ciencias exactas dentro del plan de estudios.

Frente a la evaluación de los anteproyectos y trabajos de grado, se tiene como observación general que, si bien los mismos son generalmente pertinentes y se ajustan a los requerimientos de formación y aplicación del conocimiento, deben mejorar en aspectos como la redacción, la rigurosidad en el proceso investigativo y el análisis de la información. Se valora el hecho que se esté sistematizando la evaluación de estos trabajos, de manera que se puedan establecer planes de mejoramiento desde la facultad.

Los árboles de problemas, y de medios y fines muestran como el mejoramiento de los procesos en investigación formativa requieren no sólo de inversión en infraestructura adecuada, sino además de la formulación de planes de estudio pertinentes, así como la participación y compromiso de la comunidad académica en general, incluyendo obviamente a las empresas. Así mismo, se evidencia la importancia de elaborar estrategias para la investigación que estén en concordancia con la realidad del contexto en que se desempeñan los ingenieros industriales del país, de manera que estos puedan adquirir competencias investigativas adecuadas con las exigencias del mercado. Lo anterior se ve 
evidenciado también en la Matriz DOFA, la cual sirve como herramienta para la estrategia de investigación que se pretenda formular.

El análisis de la matriz de valoración de anteproyectos y proyectos de grado elaborado por el comité curricular del Departamento de Producción en el semestre 2018-1, muestra los siguientes resultados: un 34,7 \% de los anteproyectos son aprobados sin modificaciones, y se tiene una nota promedio de valoración de 4.0 (en una escala de 0 a 5); los proyectos que son aprobados con modificaciones $(65,3 \%)$ presentan falencias en aspectos como la definición del problema de investigación, formulación de objetivos, metodología mal definida, redacción en general, manejo de normas de citación, poca profundidad del marco teórico y cronogramas de trabajo mal diseñados.

En cuanto a los proyectos de grado terminados, se tiene una nota promedio de 4,3. Dichos proyectos fueron evaluados en estos aspectos: planteamiento del problema, antecedentes, objetivos, justificación, marco teórico, metodología, resultados esperados, cumplimiento de objetivos, conclusiones y presentación. Las observaciones generales que se hicieron a los trabajos tienen que ver con aspectos relativos a la redacción, el análisis de los resultados obtenidos y su validación, el uso de referencias y la descripción de la metodología a emplear. Se hizo además un análisis de la coherencia entre la valoración del anteproyecto y la del proyecto de grado terminado, dando como resultado una coherencia promedio del $88 \%$ entre ambas valoraciones.

La Matriz de análisis de implicados muestra la importancia de hacer una gestión adecuada en el área de docencia, coordinación y relación con las empresas; una adecuada gestión de todos los implicados en un proyecto es parte fundamental para el éxito de este.

\section{DISCUSIÓN Y CONCLUSIONES}

El proceso de investigación formativa en la I.U Pascual Bravo se ha venido fortaleciendo en los últimos años, gracias en gran parte al aumento de la inversión y la implementación de políticas institucionales en investigación. Esto se ve reflejado en la percepción de la mejora de la calidad de los programas académicos que, para el caso específico de ingeniería industrial, es ratificado por los empresarios, docentes, estudiantes y egresados del programa. Además, se tiene un aumento significativo en la producción científica de los grupos de investigación; en particular el semillero SEPROCA, adscrito al programa, ha aportado de manera considerable al grupo de investigación QUALIPRO a partir de las intervenciones realizadas en diversas empresas del Área Metropolitana del Valle de Aburra.

Esta mejora en la calidad de la prestación del servicio educativo y los procesos investigativos ha tenido un impacto positivo en el entorno, el cual se ha medido a partir de aspectos como la valoración de las prácticas profesionales de los egresados del programa de ingeniería industrial de la institución en cuestión; frente a esa temática se puede leer el trabajo de Rojas, Vélez, Durango, Díaz y Rodríguez (2020) quienes proponen un análisis de la percepción del proceso de formación por competencias a partir de un estudio de percepción de la calidad de las practicas empresariales realizadas en el año 2019 por practicantes de ingeniería industrial y en donde por medio de un análisis estadístico muy riguroso se concluye entre otras cosas que las empresas le dan una alta calificación a los estudiantes por su capacidad analítica y facilidad para plantear soluciones innovadoras ante las problemáticas reales que se presentan en una planta de producción. 
En este misma línea, Pin y López (2019) hablan de la importancia de la relación entre la universidad y su entorno, dado que se espera que los resultados del proceso educativo se transfieran de forma efectiva a la sociedad, de tal manera que se potencie el desarrollo tanto económico como humano. De allí que la relación entre universidad y empresa sea vital para validar los procesos formativos en educación superior en la medida en que den cuenta de su pertinencia. Para Ferreira, Mena, Acosta y Mena (2019) la empresa se convierte en un contexto especial al convertirse en una extensión del aula de clase para un estudiante de ingeniería; las empresas validan el conocimiento teórico que da la academia, pero a su vez aporta en el desarrollo de competencias que solo son posibles de adquirir en la práctica.

Los proyectos de aula se han convertido en una herramienta adecuada para fomentar la cultura investigativa en la institución; su enfoque hacia la aplicación del conocimiento para la solución de problemas específicos en las empresas ha permitido hacer del aprendizaje algo significativo, al tiempo que mejora el nivel académico. Esta estrategia se puede potencializar en la medida en que se hagan transversales los proyectos dentro del plan de estudios, de manera que el conocimiento aplicado en cada asignatura se relacione directamente, y al final el producto entregado pueda dar fe de todo el proceso formativo. Este aprendizaje significativo ha permitido a través de los proyectos de aula ha hecho que los procesos formativos sean mas pertinentes, dado que se enfocan en la solución de problemáticas de la sociedad; en este sentido Rentería, Hincapié, Hincapié y Rojas (2019) proponen un modelo de coordenadas curriculares para la educación superior planteando una ruta metodológica que permita la generación de las competencias necesarias para los profesionales en un contexto como el actual, enmarcado en la denominada Industria 4.0.

Los semilleros de investigación como estrategia pedagógica han servido para fortalecer el proceso de investigación formativa en la Facultad de Producción y Diseño de la I.U Pascual Bravo; el semillero SEPROCA particularmente, ha permitido a los estudiantes y egresados que participan en él, afianzar los conocimientos adquiridos en su proceso de formación, adquiriendo competencias investigativas que les ha permitido un mejor desempeño laboral. De igual manera, se ha mejorado notablemente la relación entre docencia e investigación, gracias a la participación de docentes en los proyectos que se adelantan en el semillero; además, dichos proyectos han servido como trabajo de grado para algunos estudiantes que han optado por esta modalidad, lo que hace que el producto final entregado sea de una mayor calidad al desarrollado normalmente en la modalidad de monografía. A lo anterior se suma la visibilidad que ha ganado el grupo a partir de la participación en distintos eventos de investigación, como los que apoya RedCOLSI y REDPROD; este último particularmente, a través del Simposio Anual de Productividad, ha propiciado a participación del semillero SEPROCA con ponencias, posters y como apoyo logístico del evento, lo cual ha permitido que los trabajos desarrollados por los semilleristas sean publicados en memorias, revistas y sean expuestos a la comunidad académica. Aportando a lo anterior, Pepper y Terán (2019) entienden la importancia de los semilleros de investigación como herramientas para reducir la brecha existente entre las practicas y estrategias pedagógicas, los planes de estudio y los procesos de investigación; los autores afirman que los semilleros se pueden convertir en catalizadores para la integración de la universidad con su entorno.

Quedan algunos aspectos por mejorar en el proceso de investigación formativa dentro de la institución; es claro que aún falta consolidar el trabajo con los semilleros de investigación y que la producción académica puede ser mayor, además se debe trabajar en la construcción de planes de estudio y micro currículos transversales y pertinentes, alineados con las 
necesidades del medio, donde la investigación sea una estrategia de formación en las aulas de clase y la totalidad de los docentes asuman un papel de tutores, que no solo transmiten información sino que asumen un rol activo como formadores. En este sentido, estrategias como los proyectos de aula, los semilleros de investigación, la metodología ABP, los estudios de caso, han sido de mucha ayuda y han permitido mejorar los procesos de formación notablemente, así que en la medida en que estas buenas prácticas se generalicen se podrán obtener mejores resultados dentro del programa. Se espera que los resultados derivados de este proyecto de investigación sirvan como insumo para apoyar futuros planes de mejoramiento.

Por último, es preciso anotar que dentro de las dificultades que se evidenciaron por parte de los estudiantes dentro del proceso de investigación formativa está la falencia de estos en competencias de lecto-escritura y análisis lógico-matemático; esta situación es recurrente en las instituciones de educación superior del país, las cuales reciben alumnos que vienen de una formación media deficiente, y deben asumir un proceso de nivelación de conocimientos que muchas veces no es exitoso, lo cual a su vez genera vacíos en la formación profesional. Frente a esto, Vargas, Hernandez y Aguilar (2019) analizan la importancia de la formación integral de un ingeniero, lo que debe entrar en concordancia con los requerimientos del sector productivo, lo cual no solo se refiere tanto a las competencias técnicas, para lo cual el análisis lógico-matematico es primordial en cualquier programa de ingeniería, así la lecto-escritura y las habilidades blandas.

\section{REFERENCIAS BIBLIOGRÁFICAS}

Berrouet, F. (2007). Experiencia de iniciación en cultura investigativa con estudiantes de pregrado desde un semillero de investigación. Medellín: Universidad de Antioquia.

(2008). Los Semilleros como espacio de iniciación en investigación. Revista Uni-Pluri/ Versidad, 8(2), 1-16.

Bolívar, R. (2013). Los modos de existencia de la estrategia de semilleros en Colombia como expresiones de la comprensión de la relación entre investigación formativa y la investigación en sentido estricto. Múltiples lecturas, diversas prácticas. EL AGORA USB, 13(2), 433-441. Doi: http://dx.doi.org/10.21500/16578031.113

Espinoza, E. R. (2016). Formación de competencias investigativas en los estudiantes universitarios. Revista Atenas, 1(33), 18-31.

Ferreira, J., Mena, J., Acosta, A. \& Mena, J. (2019). La empresa, contexto esencial del proceso de formación profesional del ingeniero. Sus potencialidades educativas. MENDIVE, 17(4), pp. 604-618.

González, J. (2008). Semilleros de Investigación: una estrategia formativa. Psychologia. Avances de la disciplina, 2(2), 185-190. Doi: https://doi.org/10.21500/issn.1900-2386

González, M. (2006). La investigación formativa como una posibilidad para articular las funciones universitarias de la investigación, la extensión y la docencia. Revista Educación y Pedagogía, XVIII(46), 101-109.

Hernández Sampieri, R., Fernández Collado, C. \& Baptista Lucio, P. (2014). Metodología de la investigación: Roberto Hernández Sampieri, Carlos Fernández Collado y Pilar Baptista Lucio (6a. ed.). México D.F.: McGraw-Hill

Jiménez, W. (2006). La formación investigativa y los procesos de investigación científico-tecnológica en la Universidad Católica de Colombia. Revista studiositas, 1(1), 45-52.

Lammas, J. (2006). Importancia de los semilleros de Investigación en la Universidad de Cartagena. Revista Palobra, (7), 137-141. 
Landazábal, D., Pineda, E., Páez, D., Téllez, F. \& Ortiz, F. (2010). Estado de arte de conceptos sobre investigación formativa y competencias de investigación. Revista de Investigaciones UNAD, 9(2), 137-152. Doi:https://doi.org/10.22490/25391887.677

Maldonado, 1., Landazábal, D., Hernandez, J., Ruiz, A. \& Vanegas, H. C. (2007). Visibilidad y formación en investigación. Estrategias para el desarrollo de competencias investigativas. Revista Studiositas, 2(2), 43-56.

Miyahira, J. (2009). La investigación formativa y la formación para la investigación en el pregrado. Revista Médica Herediana, 20(3), 119-122. Doi:https://doi.org/10.20453/rmh.v20i3.1010

Molina, J., Martinez, L., Marín, A., \& Vallejo, E. (2012). El semillero de investigación como una estrategia para la creación de aprendizaje autónomo en la Facultad de Medicina. Medicina UPB, 31(2), 212-219.

Molineros, L. (2009). Orígenes y dinámica de los semilleros de investigación en Colombia. La Visión de los Fundadores. Popayán: Universidad del Cauca.

Osorio, M. (2008). La investigación formativa o la posibilidad de generar cultura investigativa en la educación superior: el caso de la práctica pedagógica de la licenciatura en educación básica con énfasis en humanidades, lengua castellana de la Universidad de Antioquia. Medellín: Universidad de Antioquia.

Ossa, J. \& Sierra, Z. (2001). Los semilleros de investigación como alternativa pedagógica y didáctica para la construcción de un espíritu investigativo. Revista Uni-Pluri/Versidad, 1(3), 57-60.

Parra, C. (2004). Apuntes sobre la investigación formativa. Revista Educación y Educadores, (7), 57-77.

Peláez, L., \& Montoya, J. (2013). Investigación Formativa e Investigación en Sentido Estricto: una Reflexión para Diferenciar su Aplicación en Instituciones de Educación Superior. Entre Ciencia e Ingeniería, (13), 20 - 25.

Pepper, K., \& Terán, J. (2019). El semillero de investigación estudiantil, como estrategia para la formación de investigadores. Polo del Conocimiento, 4(11), pp. 4-20.

Pin, E., \& López, R. (2019). La universidad y su relación con el entorno. Tendencia en su desarrollo. Ciencia Universitaria, 17(1), pp. 1-35.

Rendón, N. (2002). La cultura de la investigación en bibliotecología: los semilleros de investigación como una alternativa de formación en el pregrado. Revista Interamericana de Bibliotecología, 25(2), 53-71.

Renteria, J., Hincapié, E., Hincapié, S. y Rojas, I. (2019). Coordenadas curriculares para la educación superior en Colombia: Objetivos de Desarrollo Sostenible (ODS), Industria 4.0 y grupos educativos. En E, Serna (Ed), Revolución en la formación y la capacitación para el siglo XXI Edición 2, Vol. II (pp. 870-876). Medellín. Instituto Antioqueño de Investigación.

Restrepo, B. (2003). Investigación formativa e investigación productiva de conocimiento en la universidad. Revista Nómadas, (18), 195-202.

Ríos, J. (2009). Semilleros de investigación: una estrategia para abordar la ciencia. Archivos de Medicina, 9, 80-83. Doi:https://doi.org/10.30554/archmed.9.1.1307.2009

Ríos, L., Mesa, A. \& Zapata, M. (2010). Investigación formativa en la Escuela de Microbiología. Revista Hechos Microbiológicos, 1(1), 75-83.

Rojas, I. (2018). Del desarrollo económico al desarrollo económico endógeno: una mirada desde el pensamiento complejo. CINTEX, 23(1), 12-23.

Rojas, I., Cardona, J., \& Gallego, J. (2017). Estrategias de desarrollo económico local para el municipio de Girardota. (ITM, Ed.) Revista CEA, 3(6), 29-45. doi:https://doi.org/10.22430/24223182.770

Rojas, M. (2009). La investigación formativa y la docencia en la universidad. Revista UIS Humanidades, 37(2), 107-122.

Rojas, I., Vélez, Ch., Durango, J., Díaz, A. \& Rodríguez, A. (2020). Percepción del proceso de formación por competencias y su relación con las prácticas empresariales: un caso de estudio. Revista Virtual Universidad Católica del Norte, (60), 46-68. Doi: https://www.doi.org/10.35575/rvucn.n60a4 
Rojas, M. \& Méndez, R. (2013). Cómo enseñar a investigar. Un reto para la pedagogía universitaria. Revista Educación y Educadores, 16, 95-108.

Rubio, M., Vila, R., \& Berlanga, V. (2015). La investigación formativa como metodología de aprendizaje en la mejora de competencias transversales. Social and Behavioral Sciences, (196), 177 - 182. Doi:https://doi.org/10.1016/j.sbspro.2015.07.037

Silva, A., Torres, M., González, P., \& Sarmiento, J. (2008). Dinámicas de los semilleros de investigación en la UMNG. Revista Facultad de Ciencias Económicas: Investigación y Reflexión, XVI, 131-149. doi:https://doi.org/10.18359/issn.0121-6805

Trujillo, R. (2007). Los semilleros de investigación, una experiencia de formación en investigación para la inclusión social. Revista Educación en Ingenieria ACOFI, (3), 22-34.

Vargas, A., Hernandez, L., \& Aguilar, A. (2019). Exploración de competencias generales del ingeniero industrial administrador requeridas por empleadores. Revista Electronica ANFEI digital, 6(11), pp. 1-8.

Velásquez, L. (2008). Las redes de investigación virtuales: propuesta de fomento y desarrollo de la cultura investigativa en las instituciones de educación superior. Revista de Universidad y Sociedad del Conocimiento, 4, 1-11.

Vila, R., Rubio, M., \& Berlanga, V. (2014). La investigación formativa a través del aprendizaje orientado a proyectos: una propuesta de innovación en el grado de pedagogía. Innovación Educativa, (24), 241-258. Doi: http://dx.doi.org/10.15304/ie.24.1586 